\title{
LOS ENFOQUES CENTRADOS EN LAS PRÁCTICAS DE LOS PRODUCTORES FAMILIARES Una discusión de perspectivas para la investigación en sociología rural
}

\author{
APPROACHES FOCUSED ON THE PRACTICES OF FAMILY FARMS \\ A discussion of prospects for research in rural sociology
}

\author{
Clara CRaviottr c.craviott@conicet.gov.ar \\ Universidad de Buenos Aires. Argentina
}

\begin{abstract}
Resumen
El propósito de este artículo es contribuir a la discusión de algunas de las perspectivas para la investigación sobre uno de los actores fundamentales de la sociología rural, los productores familiares. Se considera el marco de surgimiento de los enfoques, así como las metodologías y dimensiones de análisis privilegiadas; sus potencialidades relativas y los vacíos o problemas por resolver. Se plantea que la noción de "estrategias de resistencia" constituye una vía de entrada interesante para el estudio de los comportamientos de este tipo de productores en el actual contexto de evolución de los sistemas agroalimentarios, porque permite dar cuenta de las contradictorias formas de vinculación establecidas, tanto con el régimen sociotécnico actualmente dominante en la producción agraria, como con los procesos de transformación experimentados por las áreas rurales, no estrictamente ligados a la cuestión agraria. Ciertos recaudos deben ser tomados, sin embargo, para no cosificar la noción de estrategia, y para no extender una lógica "resistencial" al conjunto de las prácticas desplegadas por los productores.
\end{abstract}

\section{Palabras Clave}

Estilos de producción; Estrategias de reproducción; Estrategias de resistencia; Medios de vida; Productores familiares.

\begin{abstract}
The aim of this paper is to make a contribution to the discussion of some of the approaches for the research on one of the key actors of rural sociology, the family farmers. The frame of emergence of these perspectives is considered, as well as the methods and analytical dimensions they privilege. Their relative strengths, gaps and problems to be solved are also evaluated. The concept of "strategies of resistance" is proposed as an interesting insight for the study of family farmers' practices, because it allows accounting for the contradictory relationships they establish with the sociotechnical regime currently dominant in agricultural production as well as with the processes of transformation experienced by rural areas that are not strictly linked to the agrarian question. Certain precautions should be taken, however, so as not to reify the notion of strategy or to allocate a "resistance" logic to the whole set of practices deployed by these producers.
\end{abstract}

\section{KEYWORDS}

Family Farmers; Farming Styles; Livelihoods; Reproductive Strategies; Resistance Strategies. 


\section{INTRODUCCIÓN}

Ha sido y es una preocupación recurrente de la sociología rural identificar y comprender las prácticas cotidianas de los productores, especialmente aquellos que se ubican dentro del universo de la producción familiar; explicar cómo estas varían en función de las dinámicas internas de las familias, los cambios en las condiciones contextuales y la influencia de grandes fuerzas sociales -como es el caso de las transformaciones en la estructura social y en las relaciones de género o intergeneracionales-. Pero también se intenta abstraer de estos comportamientos tendencias más generales de cambio de los sistemas agrarios y de la sociedad rural; en otras palabras, se intenta responder a la pregunta acerca de qué nos dicen tales prácticas sobre los sujetos sociales centrales del agro y sus modos de vinculación con otros actores -tanto rurales como urbanos-. La importancia de estas cuestiones deviene central en el nuevo milenio, en función de las encrucijadas que se nos plantean entre las distintas funciones del medio rural productivas, ambientales, recreativas, residenciales-, y de los destinos posibles de la actividad agraria que exceden lo alimentario (producción de insumos industriales y de biocombustibles).

Diferentes enfoques, con variable contenido y pretensión teórica, han sido pergeñados con este propósito: la aproximación centrada en los medios de vida, la vinculada a los estilos de producción y la de estrategias de reproducción, son particularmente centrales y serán abordadas en este artículo. Un aspecto común a ellas es que visualizan a los sujetos como actores. Excepto en el segundo caso, no son exclusivas de la sociología rural: la perspectiva de medios de vida ha sido ampliamente empleada en los estudios de pobreza, mientras que la de estrategias ha sido aplicado a una vastedad de grupos sociales, aunque en su variante "estrategias de supervivencia" tiene particular expresión en los trabajos sobre sectores populares urbanos. Sin agotar una revisión que no pretende ser exhaustiva, podemos visualizar asimismo que tanto el enfoque de medios de vida como el de estilos de producción tienen una fuerte impronta de investigadores de origen anglosajón, si bien han sido apropiados y empleados por otros de origen latinoamericano.

En los apartados que siguen presentaremos sintéticamente las argumentaciones centrales de los enfoques enunciados; haremos referencia a los contextos particulares en los cuales surgen, así como a las metodologías y dimensiones de análisis privilegiadas; nos preguntaremos por el carácter de las vinculaciones establecidas entre los planos micro y macro, y por el papel que juega la dimensión temporal en el análisis. También intentaremos dar cuenta de las potencialidades relativas de las diferentes líneas de pensamiento, conjuntamente con sus vacíos o problemas por resolver. Para ello consideraremos trabajos representativos de cada una de ellas y las visiones de otros investigadores. Dado que el propósito fundamental es contribuir a la discusión de algunas de las perspectivas más promisorias para la actual investigación en sociología rural, brindaremos una mayor dedicación al segundo de los enfoques mencionados -el de estilos de producción- que es precisamente el único específico de la disciplina. 
Finalizaremos planteando una noción, la de estrategias de resistencia, que constituye una vía de entrada interesante para el estudio de algunos de los comportamientos de los productores familiares en el presente contexto socioproductivo ${ }^{1}$. Se sostiene que su análisis nos permite dar cuenta de ciertas formas de vinculación que estos sujetos establecen tanto con el régimen sociotécnico actualmente hegemónico a nivel agrario como con los procesos de transformación experimentados por las áreas rurales, no estrictamente ligados a la evolución del agro. Ciertos recaudos deben ser tomados, sin embargo, para no cosificar la propia noción de estrategia, y para no extender una lógica "resistencial" al conjunto de las prácticas desplegadas por estos productores. De hecho, la investigación empírica revela que algunas de ellas pueden apuntar a la integración más que al cuestionamiento, aspecto implícito en la idea de resistencia.

\section{EL ENFOQUE DE MEDIOS DE VIDA}

Este abordaje, conocido como livelihoods approach, hace hincapié en las acciones a través de las cuales los actores rurales responden al cambio global y a las condiciones estructurales (naturales, sociales y culturales) a partir de la utilización y combinación de distintos activos (assets) a los cuales tienen acceso (Rodríguez Bilella y Tapella 2008). Como tal resulta heredero de los estudios centrados en las estrategias de los hogares conducidos en los años 80 -ya sea los de corte económico sobre la asignación del tiempo de los miembros familiares, o sociológicos, interesados en los comportamientos adoptados con vistas a la supervivencia (de Haan y Zomers 2005) - . Sin embargo, adopta un punto de vista más optimista, al invertir la visión de los pobres como víctimas y carentes de recursos, tomándolos como sujetos activos y capaces de construir con los medios de vida de que disponen, diferentes estrategias para enfrentar las situaciones que les afectan.

El desarrollo del enfoque puede ubicarse en los años 90 , en el contexto de la implementación de políticas neoliberales que preconizaban la apertura externa, el ajuste fiscal y la desregulación de las economías, y en donde las políticas sociales adquirieron un fuerte componente compensatorio. Si bien su filiación teórica es variada, indudablemente el trabajo de Sen (1981) fue una de sus principales fuentes de inspiración, con su foco en lo que las personas son capaces de ser y hacer (capabilities) en función de sus propios valores y objetivos y las oportunidades/restricciones del entorno. También puede advertirse su cercanía con la perspectiva teórica más amplia del período, que marca una vuelta a la agencia humana (Giddens) frente al estructuralismo (Niederle y Grisa 2008).

\footnotetext{
${ }^{1}$ Cuando nos referimos a este colectivo consideramos un heterogéneo conjunto de productores que engloba a campesinos y comunidades de pueblos originarios así como a sujetos que, si bien se basan de manera central en el trabajo de los miembros de la familia para la ejecución de las tareas físicas y de gestión, han accedido a niveles de capitalización que se expresan en inversiones productivas y en la posibilidad de contratar trabajo ajeno, si bien de manera no predominante.
} 
De alguna manera las circunstancias bajo las cuales surge y es empleada esta perspectiva - en íntima vinculación con la formulación de programas sociales financiados por organismos y agencias internacionales- condicionaron su pretensión teórica. No existe así un interés manifiesto por analizar las relaciones existentes entre el plano micro y el macro. La idea central es que, más allá de los condicionantes estructurales, un análisis cuidadoso de las estrategias de los pobres puede sugerir las formas en las que las "grietas" del modelo pueden ser aprovechadas, ya sea por ellos o por las organizaciones que los apoyan. Desde este marco interpretativo se hace alusión a las formas en las que las estructuras sociales más amplias y las instituciones del mercado, el Estado y la sociedad civil afectan a las posibilidades de acceder, reproducir y acumular activos; sin embargo, y tal como lo reconoce unos de sus proponentes, la distribución socialmente diferenciada de los recursos no recibe toda la atención que debería (Bebbington 2008). En la práctica los estudios carecen de un análisis de la influencia de la dimensión del poder y de las relaciones de clase en las situaciones de pobreza. Uno de sus argumentos es que las líneas entre grupos no son rígidas: inclusión y exclusión pueden variar según la esfera de la vida social considerada (De Haan y Zoomers 2005; Kay 2005).

Relacionado con lo anterior, desde el punto de vista metodológico se privilegia la investigación a escala local y los estudios de caso, aunque se sostiene que el marco de análisis debiera contribuir a captar las relaciones al interior del hogar, y de éste con la economía y las organizaciones en diferentes niveles (Bebbington 1999). El análisis comienza con la identificación de la variada gama de activos de los hogares: el capital humano (educación, habilidades, salud, tiempo), el capital social (las redes familiares, comunitarias y sociales), el capital natural (tierra, agua, bosques, etc.), el capital producido (tanto activos físicos como financieros) y el capital cultural (recursos y símbolos a disposición, como consecuencia de las estructuras sociales en las cuales el sujeto está inmerso) para abordar luego el uso que se hace de los mismos (Bebbington 2004).

Esta "multidimensionalidad" es, quizá, la mayor potencialidad del enfoque. Se visualiza a la pobreza como un fenómeno que además de aspectos económicos tiene características sociales, políticas y culturales, y se toman en consideración una diversidad de comportamientos, incluyendo algunos que a primera vista podrían ser calificados como irracionales. Además, algunos de estos trabajos tienen también una visión amplia acerca de las aspiraciones y expectativas de las personas: por medio de sus prácticas y estrategias de manejo de recursos. Éstas no solo buscan generar un ingreso material, sino también dar significado a su vida y ampliar sus capacidades de poder actuar, ya sea con vistas a la reproducción o al cambio de las reglas que gobiernan el control de los recursos. Con esta mirada sobre la pobreza y los medios de vida, Bebbington (1999) establece una conexión clara con Sen y Giddens, y también con Habermas, al considerar a los activos como vehículos de acción "instrumental" (ganarse la vida), "hermenéutica" (hacerla significativa) y "emancipadora" (desafiar las estructuras bajo las cuales se gana la vida). Estas consideraciones ayudan también a explicar las elecciones efectuadas por las personas en términos de sustitución de unos activos por otros. 
Sin embargo, resulta evidente el sesgo economicista de la perspectiva, que visualiza a la sociabilidad y la cultura como activos. Esta orientación mantuvo firmemente a la discusión en el terreno del análisis económico, aun cuando trabajos englobados dentro del mismo enfoque enfatizaron la idea de las instituciones y organizaciones como mediadoras de las estrategias de vida, lo cual requería de abordajes de corte cualitativo (Scoones 2009).

Por otra parte, su aproximación a las prácticas de los hogares carece de una apreciación rigurosa de los procesos sociales por varios motivos: en primer lugar, no reconoce que la movilización de recursos es selectiva, pudiendo favorecer la inclusión de algunos agentes al tiempo que da lugar a la exclusión de otros. En segundo término, otorga un énfasis excesivo al capital social por sobre los demás capitales, puesto que de este dependería la capacidad de los agentes de acceder a otros activos y alterar las relaciones de poder. De esta manera, pasa por alto que la participación en redes sociales tiene sus costos, especialmente para las familias pobres (González de la Rocha 1999). En este sentido, puede plantearse que se simplifican las condiciones de producción de los recursos cuya importancia se pretende destacar, al adoptarse una postura que resalta los aspectos positivos de la sociabilidad, sin considerar otras posibilidades involucradas en las relaciones sociales (Craviotti 2010).

Las falencias para lidiar con los procesos de globalización económica y los cambios en las formas de intervención estatal, así como con los desafíos de la sustentabilidad ambiental a largo plazo (considerados por esta perspectiva como factores contextuales) explicarían, según Scoones (2009), cierta pérdida de preeminencia del enfoque en relación con el de los años 90 . En este sentido, una mayor densidad en los análisis podría lograrse a partir de la incorporación de la rica tradición de estudios sobre la evolución de los sistemas agroalimentarios y la transformación del mundo rural.

\section{EL ENFOQUE DE ESTILOS DE PRODUCCIÓN}

Abordaremos ahora el concepto articulador de una perspectiva cuyo campo de aplicación específico es el de la sociología rural. Desarrollado por Jan Van der Ploeg de la Universidad de Wageningen a principios de los 90 -quien a su vez se basa en los trabajos de Hofstee producidos en los años 40 y en su contemporáneo Long- el concepto de estilos de producción (farming styles) surge como parte del clima de época que revitaliza la investigación centrada en los actores. No obstante, en el caso particular de la disciplina, el enfoque se expresa como contrapunto a la perspectiva de la "sociología de la agricultura" desarrollada en Estados Unidos en los años 80 a partir de la recuperación del marxismo clásico, en algunos casos combinado con los aportes de Chayanov (Schneider y Niederle 2008).

En términos generales, los enfoques centrados en el actor procuran explicar las respuestas diferenciales a circunstancias estructurales similares, y captar la multiplicidad de 
racionalidades, deseos y prácticas, incluyendo los asociados con el instrumentalismo. Una idea fundamental es la de "agencia", que atribuye al actor individual la capacidad de procesar la experiencia social y diseñar formas de lidiar con su vida aún bajo las formas más extremas de coerción. Siguiendo a Giddens, Long (2007) postula que dentro de los límites de información, incertidumbre y otras restricciones, los actores poseen la "capacidad de saber" (knowledgeability) y la "capacidad de actuar" (capability). Es posible agregar que, en el pensamiento giddensiano, la posibilidad de que los sujetos ofrezcan razones y narren sus acciones da cuenta de su reflexividad, aunque esta no sería total, pues los sujetos lo hacen sobre la base de conocimientos tácitos.

En este orden de ideas, la interpretación teórica de la acción social debe también abarcar los sentimientos, las emociones y percepciones, y tener en cuenta que en cualquier punto del tiempo, las personas podrían haber actuado de otra manera, ya sea positivamente, como intervenciones "intentadas", o negativamente, en términos de resignación. Long también advierte sobre la necesidad de considerar que las capacidades de saber y hacer varían según las culturas.

Objetando posibles críticas al enfoque, en el sentido de ser metodológicamente individualista (por ejemplo, por intentar explicar la conducta social en términos de las motivaciones, intenciones e intereses individuales), Long sostiene que las personas y sus ambientes están constituidos con igual correspondencia, pero "no responden con simpleza a los imperativos de marcos y normas culturales, 0 a los dictados de discursos dominantes" (Long 2007:27). Ello no implica desconocer la medida en que las acciones están encarnadas en relaciones sociales más amplias. Pero frente a las posiciones deterministas, lineales y externalistas del cambio social sostiene que todas las formas de intervención externa se "introducen" necesariamente en los modos de vida e intereses de los grupos sociales afectados, y de esta manera "son mediadas y transformadas por estos mismos actores". En última instancia, entonces, reside en ellos la capacidad de actuar, aunque importan las relaciones sociales para que la acción se haga efectiva² ${ }^{2}$.

Así como los enfoques centrados en el actor procuran explicar las respuestas diferenciales a circunstancias estructurales similares, el de estilos de producción procura captar la heterogeneidad a nivel agrario. Surge en un contexto signado por el debate sobre la mercantilización, donde la visión predominante era que los productores estaban a merced de un proceso creciente y lineal en esta dirección, que suponía atribuir valor de intercambio a una serie de esferas de la vida. Según Long (2007) los análisis de corte estructural no consideran el papel activo de los agricultores en el proceso (incluyendo

\footnotetext{
${ }^{2} \mathrm{Al}$ mismo tiempo, Long subraya algunas de las limitaciones de varios planteamientos orientados al actor promovidos en los 60 y 70 , ya sea porque adoptaban un punto de vista voluntarista de la toma de decisiones, prestando insuficiente atención al modo en que las opciones individuales están influidas por marcos más amplios de significado y por la distribución del poder y recursos; por su individualismo metodológico o porque adoptaban un modelo de elección racional basado en un número limitado de axiomas, como es el caso del de maximización de la utilidad.
} 
su resistencia a él) ni cómo se relacionan entre sí los elementos mercantilizados y no mercantilizados. De este modo, no abordan de forma satisfactoria las variaciones en las respuestas ni las estructuras intermedias entre el agricultor y el contexto más amplio. Su postura es que este retiene cierto grado de independencia en la toma de decisiones -autonomía relativa-, porque continúa controlando las maneras en que organiza su trabajo y utiliza diversos factores de producción no mercantilizados (el caso más claro es el trabajo familiar no remunerado).

Esta idea está fuertemente presente en Ploeg (1993), quien argumenta que tanto el mercado como la tecnología constituyen "espacios de maniobra" por parte de los productores, y que la manera en que una explotación agraria se relaciona con estas dimensiones varía enormemente. Existirían así grados diversos de mercantilización y el proceso como tal no es uniforme ni lineal.

En su definición inicial, la noción de estilos de producción involucra una forma específica de organizar la unidad, que en parte es moldeada por el repertorio cultural (un compuesto de ideas normativas y estratégicas acerca de cómo debería llevarse a cabo la actividad agraria), que es puesta a prueba, afirmada y si es necesario ajustada a través de la práctica (Ploeg 1993) ${ }^{3}$. Definiciones posteriores acentúan la idea de estilo como conjunto de estrategias imbuidas de una lógica inmanente -al aludir a que es una forma de organización hecha de manera coherente, cognoscible y orientada a metas-e incluyen otras dimensiones que exceden la propia organización del proceso productivo -al indicar que implica combinar tanto recursos materiales como simbólicos y sociales-. Se mantiene no obstante la idea de que cada estilo de producción se traduce en un conjunto particular de relaciones con los mercados y la tecnología (considerados como los principios estructurantes fundamentales), diferente de otras formas de ordenamiento -o estilos- posibles- ${ }^{4}$. Se argumenta también que los estilos pueden cambiar (dado que los repertorios culturales cambian), pero que no lo hacen de un día para el otro, ya que por su naturaleza tienden a evitar modificaciones radicales.

En esta breve revisión se pone de manifiesto que la relación entre los planos micro y macro es resuelta por el enfoque de estilos de producción de manera parcial. En

\footnotetext{
${ }^{3}$ En el mencionado trabajo considera cinco dimensiones de heterogeneidad: 1) El tipo de conexión con el ecosistema local (dependencia del uso y reproducción de recursos provenientes del ecosistema); 2) El tipo de intensificación (basada en el aumento gradual de la cantidad y calidad de trabajo, o en la creciente aplicación de los avances científicos a la agricultura); 3) El tipo de vinculaciones con el resto de la cadena; 4) El tipo de relaciones al interior de la unidad (distribución de las tareas según género y edad), y 5) El nivel de inserción en otras ocupaciones (pluriactividad).

${ }^{4}$ Una recuperación de las diferentes definiciones empleadas por el autor puede verse en Ploeg (2010). En este trabajo también se acentúa la vigencia del enfoque en una coyuntura de crisis mundial que provoca la reemergencia de visiones deterministas, caracterizadas por destacar la importancia de las presiones externas a la agricultura y pasar por alto la importancia de las respuestas generadas a nivel micro. Cabe indicar que el apartamiento de las formas de determinismo constituye una preocupación recurrente en sus trabajos, lo mismo que en los de Long.
} 
alguna medida se asume que los mercados y la tecnología son "fuerzas externas" a los productores, pero no se hace referencia explícita -como sí lo hace Long en el trabajo antes mencionado- a los actores que en diferentes escalas entran a jugar como mediadores de estas fuerzas y en la internalización de estas por parte de los productores ${ }^{5}$. La conexión entre la familia productora y la sociedad se daría a través de la noción de repertorio cultural; sin embargo, no se alude de manera explícita al rol que desempeñan las instancias asociativas de tipo formal o informal. Asimismo se argumenta que los estilos de producción son compartidos por un grupo amplio de productores, pero como el propio autor nos aclara, esta idea deja algunas cuestiones abiertas, como cuántos estilos hay en una región o época, o qué es lo que puede considerarse con propiedad como grupo amplio.

En un todo de acuerdo con los enfoques basados en el actor, que destacan la importancia de los escenarios en pequeña escala para entender los fenómenos macro, la investigación en estilos de producción se focaliza en regiones específicas. En su trabajo de 1993, Ploeg refiere que las indagaciones suelen hacer uso de las metáforas (0 "etiquetas") que los propios productores emplean para tipificarse y distinguirse de los demás. En su trabajo de 2010 menciona que la metodología más empleada ha sido el análisis discriminante (de componentes principales) o el análisis de clusters, que explora los patrones de coherencia que subyacen a la covariación entre variables ${ }^{6}$.

Sin embargo, un problema no previsto -relacionado no con el enfoque en sí, sino con su "apropiación" por parte de ámbitos político-institucionales- sería su utilización como método de clasificación de los productores, lo que habría dado lugar a políticas menos inclusivas (Ploeg 2010). En otras palabras, y tal como lo revela la sociolingüística, el mero acto de etiquetar no es neutro, ni en términos teóricos, ni desde el punto de vista de la intervención a través de políticas o programas.

\footnotetext{
${ }^{5}$ Por ejemplo, en términos generales los Estados han tendido a promover la incorporación de tecnologías "modernas"; pero también existen programas estatales — u organizaciones que intervienen en la implementación de estos programas-que promueven estrategias alternativas.

${ }^{6}$ No obstante, una revisión de distintos trabajos da cuenta de la multiplicidad de técnicas utilizadas. Por ejemplo, Howden et al. (1998) emplearon la técnica de grupos focales y la posterior consulta a un panel de expertos para identificar los estilos de producción vigentes en el sudeste australiano. Leeuwis realizó entrevistas cualitativas a productores lecheros de los Países Bajos, en las que les solicitó que distinguieran diferentes estilos de producción a partir de un esquema básico empleado como disparador; con los "retratos" así obtenidos realizó una encuesta donde los productores debían señalar su grado de identificación con cada uno de ellos según una escala; finalmente, empleó la técnica de análisis discriminante para reclasificar a los productores (Vanclay, Mesiti y Howden 1998). Otra variante de esta metodología es requerir a los productores que manifiesten su identificación con distintos enunciados, donde los resultados son correlacionados mediante análisis factorial (Fairwether y Klonsky 2009). En cambio, Conterato, Schneider y Waquil (2010) para el sur de Brasil emplearon el análisis factorial y el de clusters a un conjunto representado por 67 variables indicativas de los diversos recursos y estrategias empleadas por los hogares, relevadas a partir de entrevistas estructuradas.
} 
Estos aspectos metodológicos han merecido la reflexión de varios investigadores. En este sentido se ha sostenido que las respuestas brindadas en materia de categorización de sí mismos y de los otros están en buena medida condicionadas por las expectativas sociales, y que los productores no son conscientes de la existencia de diferentes estilos; sino que, más bien estos constituyen una construcción externa (Vanclay et al. 2006). También se enfatiza el rol de los investigadores en el planteamiento de las consignas disparadoras de los grupos focales, la formulación de los enunciados, o la construcción definitiva de los "retratos" con los cuales los productores deben autoidentificarse (1998). Frente a ello se sostiene que inevitablemente toda investigación parte de, o está influenciada por, una perspectiva teórica (Fairwether y Klonsky 2009).

Otra cuestión mencionada es que por lo general los trabajos minimizan los roles diferenciales de los miembros de los hogares, producto de una metodología que no tiene en cuenta la cuestión de género, ya que, por lo general, se entrevista a productores y no a productoras (Vanclay et al. 2006). A esto mismo se puede agregar la falta de consideración de aspectos generacionales. Sin embargo, el pluralismo metodológico a que ha dado lugar la investigación inscrita en esta corriente revela que no necesariamente se deriva de ella una estrategia metodológica determinada, o que no puedan incorporarse las perspectivas y visiones del conjunto de los miembros del hogar en los estudios ${ }^{7}$.

En realidad, son las cuestiones teóricas las que ameritan, quizá, un mayor análisis si se decide optar por esta perspectiva de investigación. Retomando lo antes planteado acerca de la relación entre los niveles micro y macro, son relativamente limitadas las dimensiones analíticas consideradas. No se recuperan los mecanismos de interacción de los productores (que incluyen intercambios no mercantilizados y aspectos no necesariamente vinculados a lo estrictamente productivo). Así como no se presta particular atención a las dimensiones sociales, se tiende a poner en un segundo plano a las simbólicas o identitarias.

Tampoco el enfoque lograría captar la coevolución con el mundo circundante, al no percibir cómo este es influenciado por las estrategias de los productores (Noe y Alroe 2003). Ploeg no habría problematizado el componente territorial del concepto de estilos, restringiéndolo a la lógica productiva de las unidades familiares (Schneider y Niederle 2008).

Al respecto, puede sostenerse que un argumento implícito en los trabajos encuadrados dentro de esta perspectiva es que los estilos de producción "alternativos" al régimen actualmente dominante en la agricultura -basado en la modernización, la ampliación de escala y una creciente mercantilización- tienden a potenciar y afirmar los vínculos de los productores con los espacios locales, dando lugar a procesos de desarrollo endógeno. Sin embargo ello debiera contrastarse empíricamente en cada caso y no asumirse como un presupuesto del análisis. Las vinculaciones locales o el grado de "anclaje" territorial de los productores también deben ser explorados en los estilos que siguen un patrón

${ }^{7}$ Ploeg (2010) señala al respecto que el enfoque no conlleva un método universalmente aplicable, pero la posición subyacente (que ve a la agricultura como una construcción social) sí lo es. 
"convencional" o suponen cierto grado de hibridación (probablemente las situaciones más frecuentes). Según Vanclay et al. (2006), Ploeg abandonó su posición de los estilos como mutuamente excluyentes y más recientemente se inclinaría a sostener que en la práctica no existen cortes abruptos entre ellos, lo que apoyaría la idea de hibridación ${ }^{8}$. Pero sin duda alguna el estilo que ha recibido mayor atención en esta literatura es el de farming economically, basado en el escaso uso de insumos comprados -propio de algunas comunidades campesinas de países en desarrollo, e indicativo de un proceso de "recampesinización" en el contexto de los países desarrollados.

Con respecto a este tema, resulta pertinente el planteamiento de Goodman (2004), cuando señala que todavía no está del todo claro qué tipo de actividades responden a la noción de estilos de producción alternativos, sugiriendo que sus proponentes se encontrarían en la disyuntiva de focalizarse exclusivamente en las formas innovadoras emergentes y una posición más abarcadora, que no enfatiza tanto la ruptura con la agricultura intensiva convencional ${ }^{9}$. Esto implicaría desbrozar los análisis concretos de los componentes de tipo normativo que pueden identificarse en algunos de los estudios encuadrados en esta perspectiva.

Frente a todos estos comentarios, puede plantearse que el enfoque de estilos de producción posee una validez intuitiva inmediata, no solo para la investigación, sino también para las actividades de extensión (Vanclay et al. 2006). Indudablemente ha sido fructífero, en tanto en cuanto ha dado lugar a estudios empíricos y reflexiones analíticas por igual, y ha generado líneas de investigación vinculadas. En retrospectiva, Ploeg (2010) identifica las siguientes: 1) la co-producción hombre-naturaleza y las innovaciones; 2) el desarrollo endógeno, especialmente en áreas marginales; 3 ) los nuevos estilos de producción caracterizados por la multifuncionalidad; y 4) la revitalización del debate campesino.

Teniendo en cuenta las objeciones principales efectuadas al enfoque, consideramos por nuestra parte que el análisis de la distribución de variables "duras" a nivel regional (tales como la escala y modalidades de tenencia de las explotaciones, los patrones de uso del suelo y de incorporación de tecnología, etc.), conjuntamente con el estudio de las combinaciones de prácticas desarrolladas por los productores y los significados atribuidos a ellas, puede revelar la multiplicidad de estilos existentes en un área en un determinado contexto histórico. Asimismo, una ampliación de las dimensiones de análisis en los trabajos empíricos puede contribuir a captar de manera más acabada los vínculos entre los hogares y diferentes ámbitos sociales, así como la existencia de

\footnotetext{
${ }^{8}$ Una cuestión explícitamente reconocida por Howden y otros (1998) y Conterato et al. (2008), a partir de los resultados de sus investigaciones.

${ }^{9}$ Agrega además que la forma en que estas estrategias alternativas pueden ser eficaces para mitigar problemas rurales de largo alcance, como es el caso de la desigualdad en los ingresos, el empleo mal remunerado y la pobreza rural, así como la cuestión del desarrollo desigual reciben una atención menor en esta literatura (Goodman 2004).
} 
esferas mercantilizadas y no mercantilizadas (primer paso para visualizar como estas se influyen mutuamente). Este tema no es una mera cuestión operacional: ¿Puede existir una "desmercantilización" del proceso productivo, conjuntamente con una creciente mercantilización de otras esferas de la vida social? Si es posible, ¿en qué condiciones? ¿qué tipo de tensiones genera? Estos son solo algunos de los interrogantes posibles.

Esta ampliación de dimensiones también permitiría dar cuenta de la agricultura como modo de vida y no solo como una forma de organizar la producción, cuestión especialmente relevante en los productores familiares. Otro tema por explorar es cómo la adopción de diferentes estilos se vincula con los procesos o trayectorias experimentadas por unidades específicas. Ello implica adoptar una perspectiva diacrónica, que contribuiría a explicar la heterogeneidad encontrada a nivel agrario. Una posibilidad auspiciosa en este sentido es el empleo de técnicas de panel en base a muestras de hogares.

\section{EL ENFOQUE DE ESTRATEGIAS DE REPRODUCCIÓN}

Reflexionando acerca de su experiencia de investigación en México, señalaba Long (2007) que la cuestión de las "estrategias" de los actores constituía un concepto central, porque le permitía comprender cómo los habitantes rurales trataban de resolver sus problemas de sustento y organizar sus recursos e implicaba que, dentro de los límites que enfrentan, éstos construyen activamente tanto sus modelos de organización de la explotación y del hogar como sus maneras de lidiar con los organismos y agencias de desarrollo. Nos introduce luego en los riesgos involucrados en la propia noción de estrategia, al sostener que:

"al enfocar de esta manera las estrategias, podría parecer que se enfatizan demasiado los procesos de cálculo racional y toma de decisiones, pero a lo largo del trabajo procuramos anclar nuestras preguntas de investigación, observaciones y análisis a las experiencias vividas de los actores, a sus deseos, entendimientos y autodefiniciones de situaciones problemáticas, intentando no imponer nuestras categorías de interpretación" (Long 2007:68).

Precisamente esta idea -la de un sujeto racional que toma decisiones sobre la base de información suficiente- es uno de los aspectos más habitualmente objetados a la noción de estrategia que retomaremos aquí.

Por una parte, un aspecto a tener en cuenta es que con frecuencia se asume un interés universal por maximizar la utilidad, pasándose por alto la presencia de otros valores que inciden en la toma de decisiones, así como la posible existencia de diferentes preferencias en los integrantes de las familias. En realidad, a partir de los aportes de los estudios de género, el reconocimiento de las tendencias a la individualización de las personas y hacia la creciente diversificación de las trayectorias vitales es posible poner en cuestión la visualización de los hogares como unidades de intereses homogéneos. La influencia de la dimensión temporal tampoco puede ser desdeñada, en tanto las personas pueden desplegar diferentes estrategias en distintos momentos de su vida. 
Por otra parte, al plantearse la "conciencia" de las intenciones se dan al menos dos problemas: muchas veces la acción social carece de propósitos deliberados y los resultados más "estratégicos" son subproductos colaterales no buscados (Arteaga 2007; Moen y Wethington 1992). Como indica Giddens la cognoscibilidad de los agentes tiene sus límites; por un lado en el inconsciente, y por otro, en las condiciones no conocidas y en las consecuencias no intencionadas de la acción (Scribano 2009). Tanto este autor como Bourdieu a través de su noción del sentido práctico nos recuerdan que gran parte de las acciones cotidianas son de carácter pre-reflexivo.

En realidad, podemos ver que buena parte de las cuestiones mencionadas tienen que ver con la atribución de sentido a las acciones desarrolladas por los miembros de los hogares y de un componente electivo, que puede ser aprehendido y verbalizado a través del discurso.

Una posible salida a estos problemas es ofrecida por Bourdieu (1994) cuando postula el concepto de estrategias de reproducción social, entendidas como el conjunto de prácticas por medio de las cuales las familias tienden -de manera consciente o inconsciente- a reproducir su posición en el espacio social y por esta vía el sistema de diferencias constitutivo de todo orden social. Como parte de su propuesta teórica más amplia, construida a partir de las nociones de "habitus" y "campo", Bourdieu apunta a que las estrategias de reproducción se encuentran enraizadas en las disposiciones duraderas internalizadas por el individuo durante el transcurso de su socialización (el habitus), que en última instancia depende de las condiciones sociales.

Ello no implica que las expectativas de los agentes repliquen mecánicamente sus oportunidades objetivas: las disposiciones son capaces de sobrevivir a las condiciones económicas y sociales de su propia producción. Además conforman un sistema "abierto" constantemente sujeto a experiencias y afectado por ellas, de una manera que refuerza o bien modifica sus estructuras (Bourdieu y Wacquant 2005:192).

El desvelamiento de las condiciones de la acción requiere entonces del análisis de las disposiciones y la posición ocupada en el campo ${ }^{10}$. Esto implica reconstruir las estructuras objetivas y reintroducir la experiencia de los agentes con el fin de explicar las categorías de percepción y apreciación que estructuran su acción. El primer momento de análisis tiene prioridad epistemológica, pues los puntos de vista de los agentes varían según el punto que ocupan en el espacio social objetivo (Bourdieu y Wacquant 2005).

Propuesta como una vía superadora del objetivismo y el subjetivismo, la noción de habitus le permite a Bourdieu resolver el dilema entre micro y macroanálisis, y entre

\footnotetext{
${ }^{10}$ Bourdieu "reconstituye" la noción de estructura desplazándose a una visión de la sociedad constituida en campos. Un campo es un espacio de conflicto y competencia en el cual los participantes rivalizan por el monopolio sobre el tipo de capital que es eficaz en él. Bourdieu lo define como una red de relaciones entre posiciones objetivamente definidas por su situación en la estructura de distribución de especies de capital, así como por su relación objetiva con otras posiciones.
} 
agente y estructura ${ }^{11}$. El tipo de racionalidad que atribuye a las prácticas se aparta de la noción de actor racional en tanto se trata de una "racionalidad" socialmente limitada por las condiciones que produjeron el habitus; es decir, lo que los agentes aprehenden en relación a sus condiciones materiales de existencia y los condicionamientos que estas les imponen, les hace delimitar lo potencialmente posible y descartar prácticas imposibles que, en rigor, son impensables (Wilkis 2004; Jiménez Zunino 2010).

Las líneas de acción sugeridas por el habitus pueden estar acompañadas de un cálculo costo-beneficio; sin embargo, es necesario echar luz sobre aquellas prácticas que son "razonables" para los sujetos, sin ser el producto de un propósito razonado y menos aún de un cálculo consciente (Bourdieu y Wacquant 2005).

La noción de habitus permite a Bourdieu explicar por qué la vida social presenta regularidades al mismo tiempo que preserva la idea de agencia: el habitus es un principio "generador y organizador" de prácticas objetivamente adaptadas a su fin, aunque sin suponer la búsqueda consciente de fines. De allí su regularidad, sin ser el producto de la obediencia a reglas.

En esta perspectiva, la noción de estrategia se basa en la construcción de una teoría de la práctica -que reemplaza la lógica mecánica de la estructura por la lógica dinámica del juego (Bourdieu 1994) -. Las estrategias se definen constantemente a partir del encuentro entre el habitus y una coyuntura particular del campo. Su análisis supone tener en cuenta las acciones anteriores para entender las actuales y reintroducir el conjunto de las estrategias desplegadas en todos los órdenes, en la medida en que constituyen un sistema "cronológicamente articulado" (1994) ${ }^{12}$. Un elemento importante al respecto son las estrategias de reconversión de capitales que apuntan a mantener la posición social.

Adentrándonos en aspectos más operacionales de las estrategias de reproducción -uno de los ejes analíticos propuestos en este artículo- se puede señalar que la perspectiva bourdesiana considera tanto la composición del patrimonio que se trata de transmitir (el peso relativo de cada especie de capital) como el estado de los mecanismos de

\footnotetext{
${ }^{11}$ Otro camino en la misma dirección es propuesto por Giddens a través de su teoría de la estructuración, al indicar que la estructura modela las prácticas, pero también las prácticas constituyen y reproducen las estructuras. De esta manera la agencia humana y la estructura no se oponen entre sí, sino que una presupone a la otra. Su concepción de los agentes como "capaces" y "conocedores" implica que estos pueden poner sus capacidades estructuralmente formadas en acción de una manera creativa; si suficientes personas o pocas personas con el poder suficiente actúan de manera innovadora pueden transformar las mismas estructuras que les dieron la capacidad de actuar (Sewell 1992). Sewell indica sin embargo algunas de las cuestiones no resueltas por el enfoque, proponiendo algunos conceptos que permitirían captar más cabalmente la transformación de las estructuras.

${ }^{12}$ Este punto es también remarcado por los investigadores de la corriente del life course (curso o trayectoria de vida), que enfatiza la naturaleza temporal de las estrategias: su timing, duración y secuencia en el ciclo familiar e individual, y su carácter acumulativo, donde las elecciones en tiempos anteriores enmarcan las actuales. Por otra parte el cambio histórico es también un ingrediente fundamental para entender las estrategias familiares (Moen y Wethington 1992).
} 
reproducción en una sociedad determinada (el mercado, los derechos, las instituciones escolares). En cuanto a las dimensiones de indagación identificadas, abarcan tanto las "estrategias de inversión biológica" (las estrategias de fecundidad y profilácticas), de "sucesión" (que buscan la transmisión del patrimonio familiar entre generaciones), de "inversión económica" (orientadas a la perpetuación y aumento del capital en todas sus formas, incluyendo en sentido amplio a las estrategias orientadas a la creación y mantenimiento de relaciones sociales útiles), las "educativas" (estrategias escolares y de inculcación de normas) y las de "inversión simbólica" (tendentes a conservar y aumentar el capital de reconocimiento social). La unidad de análisis es siempre la familia actuando como sujeto colectivo, aunque de todas maneras es necesario conocer la relación de fuerzas entre sus integrantes.

La potencialidad de este enfoque radica en reparar en que la importancia de tales dimensiones varía en función del "lugar" que adquieren ciertos dominios fundamentales en cada ámbito social particular, que se traducen en diferentes propensiones a invertir en diferentes instrumentos de reproducción. Ya que evidentemente no es lo mismo una sociedad donde pesan las estructuras del mundo económico y del Estado, que una donde la comunidad cobra importancia central. Además, desde esta perspectiva se reconoce que toda estrategia desempeña al mismo tiempo una función de inclusión y otra de exclusión.

Este enfoque asume el rol de los sujetos en su construcción sin desconocer aspectos estructurales. Sin embargo, su riesgo es que al poner énfasis en la reproducción del orden instituido puede conducir a un callejón sin salida a la hora de explicar el cambio social. El "reproductivismo" ha sido una de las críticas más habituales al pensamiento de Bourdieu, al que se le atribuye poca apertura para estudiar los procesos de resistencia (Alonso 2004). En este sentido se sostiene que los actores sociales serían capaces de aplicar un repertorio de esquemas culturales mucho más vasto que los habitus universalmente homólogos planteados por Bourdieu, y tendrían acceso a conjuntos de recursos también heterogéneos (Sewell 1992).

Justo es señalar, sin embargo, que Bourdieu ha considerado esta cuestión, si bien no con la misma centralidad, al indicar que aquellos que monopolizan el capital específico de un campo se inclinan hacia estrategias de conservación, mientras que los que disponen de menos capital, hacia estrategias de subversión (Scribano 2009). Asimismo los agentes pueden intentar transformar las reglas de juego de un campo, mediante prácticas que apuntan a cambiar el valor relativo de cada especie de capital (Bourdieu y Wacquant 2005).

\section{LAS ESTRATEGIAS DE RESISTENCIA EN LOS PRODUCTORES FAMILIARES}

Con estos aportes en mente, retornamos ahora al campo de la sociología rural para abordar una expresión particular de las estrategias de reproducción que también pone énfasis en la cuestión de la agencia, pero al mismo tiempo nos remite claramente a 
una contraparte, con poder o capacidad de control frente a la cual el sujeto se opone: la noción de estrategias de resistencia. Así, esta implica incorporar un matiz diferencial con respecto a la connotación neutra presente en la idea de estrategias de reproducción.

Si bien las referencias empíricas englobadas bajo los términos "resistir" o "resistencia" abundan, especialmente en los estudios sobre sectores populares, pocas veces se discuten sus implicaciones teóricas. Un antecedente en la materia es el conocido trabajo de James Scott (2000), que hace hincapié en la importancia de las formas de resistencia desplegadas tanto en el plano material como simbólico a través de las cuales los sujetos subordinados resisten la dominación ${ }^{13}$.

En esta perspectiva ambas formas de resistencia -material y simbólica- se complementan y apoyan mutuamente. Desarrollado inicialmente a partir del estudio de un poblado malayo que experimentaba las transformaciones disruptivas de la Revolución Verde, el aporte de Scott nos llama la atención hacia las formas individuales no planificadas y anónimas de la resistencia campesina, que engloba todos los actos orientados a mitigar o negar los requerimientos efectuados por otras clases, o encaminados a promover sus reivindicaciones (Scott 1987). Cabe observar que esta definición elude el requisito de la acción colectiva, lo que no quiere decir que las acciones en cuestión no estén enraizadas en una cultura popular común y en redes sociales densas que posibilitan una cooperación tácita o complicidad entre los actores. La relativa "seguridad" de estas formas de resistencia en gran medida se vincularía con su pequeña escala, no obstante ello, su carácter acumulativo puede generar eventos de consecuencias más significativas (1989)

En Ploeg (2007) se establecen puntos de contacto con esta visión y se argumenta también que el concepto de resistencia se encuentra más extendido en la actualidad de lo que habitualmente se supone: no solo engloba las formas abiertas de disputa y las manifestaciones camufladas de las que habla Scott en su trabajo, sino también un campo de acción más amplio y probablemente más importante que es el de los "espacios de producción". Se refiere de este modo a un conjunto de prácticas cada vez más interrelacionadas, a través de las cuales el campesinado se constituye como sujeto diferente; activamente continuadas o recreadas para confrontar los modos de ordenamiento actualmente dominantes ${ }^{14}$.

Diferenciándose de la concepción tradicional del término, derivada de la física, Ploeg sostiene que la resistencia no es más una forma de reacción sino de acción basada

\footnotetext{
${ }^{13}$ Dentro de las primeras ubica ciertas acciones como la caza furtiva, el absentismo en el trabajo, el sabotaje, la evasión de impuestos y la ocupación clandestina de tierras. Mientras que las segundas se hallan presentes de manera encubierta en prácticas tales como los rumores, los cuentos y fiestas populares y otras modalidades simbólicas que conforman un "discurso oculto". Representan una crítica del poder efectuada a espaldas del dominador para evitar los riesgos de una confrontación abierta (Scott 2000).

${ }^{14}$ Aunque en un todo de acuerdo con su perspectiva que pone énfasis en la dimensión de análisis micro, sostiene que una característica de estas prácticas es que involucran la búsqueda de soluciones locales a problemas globales
} 
en la innovación. Involucra la búsqueda de autonomía a través del desarrollo de un conjunto de recursos auto-gobernados, tanto naturales como sociales (conocimiento, redes sociales, instituciones) ${ }^{15}$.

Es importante recuperar el contexto particular en que se plantea este concepto de estrategias de resistencia, que es el de la globalización de la agricultura y de instauración de un régimen sociotécnico de características "productivistas", que involucra el incremento de escala, la industrialización de la producción, y una creciente dependencia del productor de los mercados de insumos, productos y factores ${ }^{16}$. Así cabría plantear que en este marco, en buena medida adverso a las formas de producción familiar en pequeña escala en tanto genera costos crecientes y fragilidad en los ecosistemas, varias de las prácticas orientadas a la reproducción -es decir, a reproducir la posición en el espacio social en términos de Bourdieu- adquieren -a veces de manera inconsciente y no planificada- el matiz diferencial de la resistencia.

El interés por la resistencia en los espacios de producción de alguna manera explica las dimensiones de análisis priorizadas por el autor. Ploeg menciona las siguientes: 1) la creación de nuevos productos y servicios, que implican nuevos circuitos mercantiles y el surgimiento de explotaciones multiproducto y diversificadas, 2) la búsqueda de una menor dependencia de los mercados de insumos (conceptualizado como estilo de farming economically), 3) la reconexión de la agricultura con la naturaleza, 4) la pluriactividad, 5) el desarrollo de nuevas formas locales de cooperación, y 6) la reintroducción de la artesanía. Sin embargo, puede plantearse que no queda claro el criterio subyacente a esta selección, y si basta con la existencia aislada de algunas de estas dimensiones para hablar de resistencia ${ }^{17}$.

Aquí es nuevamente válida la observación de Goodman (2004), cuando problematiza cómo se visualizan los vínculos con la agricultura "convencional". La propuesta de investigación será diferente si se los ve en términos de ruptura radical o bien como una combinación/hibridación de elementos. Esto último constituye una posición más abarcadora y menos orientada a la búsqueda de casos paradigmáticos -visualizados como base de la transición hacia un régimen "moralmente" superior-.

${ }^{15}$ Un punto particularmente interesante que agradecemos a un evaluador anónimo de este artículo (aunque por razones de espacio no lo exploraremos aquí), se refiere a las condiciones que hacen posible el surgimiento de estas acciones de resistencia. El desarrollo de prácticas alternativas no solo sería consecuencia de las decisiones individuales de los agricultores, sino que se vería favorecida por su participación en redes e interrelación con otros actores, no solo locales sino también externos.

${ }^{16}$ De acuerdo con Rip y Kemp (1998), en Ploeg et al. (2004), un régimen sociotécnico es el conjunto de reglas comprendidas en un complejo coherente de conocimiento científico, prácticas ingenieriles, tecnologías del proceso productivo, características del producto, habilidades y procedimientos, maneras de manejar artefactos y personas y formas de definir los problemas. Prescribe las prácticas agrícolas de manera directa 0 indirecta e implica una trayectoria específica de investigación y desarrollo.

${ }^{17}$ Tal vez la apreciación de Ploeg esté fuertemente imbuida de los trabajos sobre transiciones en los regímenes sociotécnicos, que introducen la noción de que los cambios radicales emergen de "nichos", en los cuales se experimenta con alternativas en pequeña escala y en los márgenes, que pueden adquirir centralidad cuando cambian las condiciones y emergen nuevas oportunidades (Scoones 2009). 
Otra posible objeción se refiere a la validez del concepto de estrategias de resistencia en el caso de sujetos cuya posición de subordinación no es tan evidente. En otras palabras, ¿la noción de resistencia se aplica a aquellas prácticas que por sus características van en dirección contraria al modelo hegemónico, o también tiene mucho que ver con la condición subordinada del sujeto que la ejerce? En este sentido pensamos que tanto campesinos como productores familiares capitalizados poseen una posición subordinada en los mercados, y por lo tanto, son pasibles de estrategias de resistencia que adquieren, sin embargo, contenidos diversos, ya que los segundos están sujetos más definidamente a las presiones modernizadores del actual régimen sociotécnico.

Algunos autores (Schneider y Niederle 2010) más que referirse al "cómo" se expresa la resistencia se refieren al objetivo al "que" se apunta: no solo es mantener cierta forma de producir, sino también recursos simbólicos y el patrimonio inmaterial de las áreas rurales. Al respecto vale la pena introducir el planteo de Alonso, Arribas y Ortí (1991:48) a partir de un estudio sobre los agricultores españoles, cuando sostienen:

"[...] la actitud básica parecería ser la de continuar siendo labrador, humilde y marginado, pero dueño y señor de su tierra, adaptándose tan solo a la función de empresario - degradando su relación con la tierra a la de un instrumento racional de producción, y aceptando un principio de cálculo rentable como principio de su actividad- de forma forzada y en la medida imprescindible para cumplir con su programa de resistencia. El denominador común de sus intereses [parece] ser preservar al máximo su autosuficiencia social y solo después económica, de modo compatible con la impuesta adaptación a las nuevas condiciones de mercado y de producción. Desde un punto de vista económico, sus cambios tendían a limitarse a los exigidos por las ineludibles medidas defensivas para mantener su tradicional status de trabajadores autónomos, con el menor deterioro posible" (la cursiva es nuestra).

Es decir, se trataría de la aceptación "instrumental" y "controlada" de ciertas pautas en pos de preservar la persistencia de la unidad productiva familiar y la continuidad de cierta forma de vida (aunque transformada).

Así, el núcleo problemático de esta perspectiva es cómo y de qué manera se atribuye a las prácticas un "sentido o lógica resistencial" al régimen sociotécnico dominante, más allá del contenido concreto que estas adoptan. Apreciaciones todas que nos remiten a recuperar la dimensión identitaria y su expresión en los discursos y representaciones, más allá de la importancia indudable que adquiere el análisis de los espacios de producción, ya que la generación de prácticas alternativas implica una construcción cultural que hasta cierto punto es compartida con otras personas. Central a ella es cómo los agentes sociales visualizan las transformaciones experimentadas por la agricultura y, en función de ello, otorgan un significado particular a sus acciones cotidianas. 


\section{REFLEXIONES FinALES}

Las diversas perspectivas delineadas en este artículo hacen hincapié -en diferentes grados- en la capacidad de acción de los sujetos frente a las estructuras, aún de quienes detentan una situación de subordinación. Sus acciones pueden tanto convalidar como apuntar a modificar el orden social instituido.

La potencialidad del enfoque de estrategias de resistencia aplicado a la investigación sobre productores familiares, en el actual contexto de evolución de nuestras sociedades, radica en que pone en primer plano el análisis de la relación establecida con el régimen sociotécnico prevaleciente -caracterizado por la ampliación de escala, la intensificación productiva y la incorporación creciente de insumos comprados, entre otros aspectos- y con los procesos de transformación experimentados actualmente por las áreas rurales en función del surgimiento de nuevas funciones y demandas sobre estos espacios, que pueden traducirse en la mercantilización de nuevas esferas de la vida social. No es posible ni adecuado pensar en estas estrategias "en abstracto", sin identificar las características del contexto social e histórico más amplio en el cual se desarrollan. En este sentido sigue siendo válida la afirmación de Bourdieu respecto a que el análisis de estrategias debe considerar el estado de los mecanismos de reproducción en una sociedad determinada.

Este enfoque, aún en construcción en la sociología rural, nos invita a pensar en la contraparte contra la cual se ejerce cierta resistencia. Tal como Scott nos amplía la visión al referirse a las formas cotidianas de resistencia, habría que pensar en las formas cotidianas de ejercicio del poder, inclusive teniendo en cuenta que diferentes niveles del Estado pueden asumir orientaciones divergentes (Sivaramakrishnan 2005).

La utilidad del enfoque en el contexto actual parece clara, teniendo en cuenta la expansión de ciertas modalidades de producción e intervención sobre los espacios rurales caracterizadas por una escasa consideración de los recursos naturales y del valor del trabajo humano, a favor de la rentabilidad económica, la flexibilidad y la rotación del capital. Desde este punto de vista la idea de estrategias de resistencia nos invita a preguntarnos si existen comportamientos que a primera vista son difíciles de explicar puesto que cuestionan la maximización de la utilidad pero se encuentran enraizados en otros valores y significados. Tal como lo plantean algunos de los proponentes del enfoque de los medios de vida, para avanzar en una sociología rural comprensiva es necesario captar la variedad de motivaciones subyacentes en los actores.

Desde esta perspectiva, las estrategias de resistencia no constituyen meras reacciones defensivas, consistentes en continuar con las mismas prácticas en un contexto diferente. Contemplan también la innovación, en buena medida orientada a potenciar aquello de lo que se dispone. Tal como surge de los párrafos anteriores, la propuesta de considerar las estrategias de resistencia en los espacios de producción debe ser complementada con la comprensión de los aspectos simbólicos, que con frecuencia representan el punto de partida y el sustento de las formas basadas en lo material. 
Sin embargo no debiera centrarse una atención exclusiva en las situaciones de ruptura, sino también estudiarse la manera en que los "ámbitos de resistencia" se combinan con otros de convivencia o aceptación instrumental de ciertos elementos propios del régimen sociotécnico dominante en aras del objetivo de preservar la condición de productores (la reproducción de la posición social).

Esto último nos introduce en aspectos epistemológicos, ya que en la misma identificación de las estrategias de resistencia radica el complejo problema de la atribución de sentidos: Si lo hace el investigador teniendo en cuenta los resultados actuales 0 potenciales de la acción emprendida o considerando los puntos de vista de los agentes. Además de los significados explicitados están aquellos que permanecen latentes, y que forman parte de disposiciones internalizadas de las que nos habla Bourdieu con su noción de habitus.

Por otro lado, las prácticas concretas también dependerán de los movimientos de aquello a lo cual se opone el sujeto que resiste. El caso de la incursión por parte de grandes empresas en el ámbito de los productos tradicionales revalorizados, transformando la lógica de producción artesanal en la cual estos productos se basan, es un ejemplo pertinente. Esta situación puede afectar a su condición "natural" de ámbito de resistencia y obligar a una redefinición de las estrategias encaradas por los productores familiares.

El riesgo de circunscribirse a lo micro -un aspecto frecuentemente cuestionado a este tipo de enfoques- puede ser evitado con la consideración de que estas prácticas dependen, para su emergencia, de disposiciones socialmente construidas y, para su desarrollo, del incrustamiento en relaciones sociales, no solo con otros agentes locales sino también extralocales. El estudio de los efectos de estas prácticas sobre el ambiente y el territorio, ya sea en función de su carácter acumulativo (aunque no necesariamente organizado como señalaba Scott), como también por la existencia de efectos sinérgicos o complementarios nos conduce, por otra vía, a superar el plano de análisis micro.

Si bien el término "estrategias de resistencia" puede aparecer problemático para referirse a productores familiares capitalizados cuya posición en el espacio social no expresa una clara subordinación como es el caso de los campesinos, su consideración nos permite poner la mirada sobre un vasto conglomerado de productores que, si bien en determinadas circunstancias pueden haber logrado la reproducción ampliada de sus condiciones de existencia, experimentan un conjunto de limitaciones en función de las formas de producir que tienden a consolidarse, junto con el discurso modernizador que las acompaña.

Por último, no queremos dejar de pensar, siguiendo a Habermas, que las acciones estratégicas no agotan el conjunto de las prácticas de los agentes sociales. Además de las acciones conscientes o inconscientes orientadas a reproducir la posición en el espacio social existen otras de un carácter completamente diferente. Nos referimos a aquellas de índole comunicativa, mediadas simbólicamente y orientadas al entendimiento, cuyo lugar deviene de nuestro carácter de sujetos sociales. 


\section{RefERENCIAS BibliográfícAS}

Alonso, L., J. Arribas y A. Ortí. 1991. "Evolución y perspectivas de la agricultura familiar: De "propietarios muy pobres" a agricultores empresarios". Política y Sociedad 8:35-69.

Alonso. L. 2004. "Pierre Bourdieu, el lenguaje y la comunicación: del análisis de los mercados lingüísticos a la denuncia de la denigración mediática". Pp. 215-254 en Pierre Bourdieu: las herramientas del sociólogo, editado por L. Alonso, E. Criado y J. Moreno Pestaña. Madrid: Fundamentos.

Arteaga, C. 2007. "Pobreza y estrategias familiares: Algunas reflexiones." Revista Mad 17:144-164.

Bebbington, A. 1999. "Capitals and capabilities: A framework for analyzing peasant viability, rural livelihoods and poverty". World Development 12:2021-2044.

Bebbington, A., S. Guggenheim, E. Olson y M. Woolcock 2004. "Exploring social capital debates at the world bank". Journal of Development Studies 40:33-64.

Bebbington. A. 2008. "La sostenibilidad social de los recursos rurales: apreciaciones a partir de los conflictos mineros en Latinoamérica." Debate Agrario 42:31-78.

Bourdieu, P. 1994. "Stratégies de reproduction et modes de domination". Actes de la Recherche en Sciences Sociales 105:3-17.

Bourdieu. P. y L. Wacquant 2005. Una invitación a la sociología reflexiva. Buenos Aires: Siglo XXI Editores.

Conterato, M., S. Schneider y P. Waquil 2010. "Estilos e agricultura: uma perspectiva para a análise da diversidade da agricultura familiar". Ensaios FEE 31:149-186.

Craviotti, C. 2010. La otra agricultura. Trayectorias y estrategias de microemprendedores pampeanos. Buenos Aires: Editorial Biblos.

De Haan, L. and A. Zoomers 2005. "Exploring the frontier of livelihoods research". Development and Change 36:27-47.

Fairwether, J. y K. Klonsky 2009. "Response to Vanclay et al. on Farming Styles: Q methodology for identifying styles and its relevance to extension". Sociologia Ruralis 49:189-198.

González de la Rocha, M. 1999. "La reciprocidad amenazada: un costo más de la pobreza urbana". Revista Latinoamericana de Estudios del Trabajo 3:33-50.

Goodman, D. 2004."Rural Europe Redux? Reflections on Alternative Agro-Food Networks and Paradigm Change". Sociologia Ruralis 44:3-16.

Howden, P., F. Vanclay, D. Lemerle y J. Kent 1998. "Working with the grain: Farming styles amongst Australian broadacre croppers." Rural Society 8:109-125. 
Jiménez Zunino, C. 2011 “¿Empobrecimiento o desclasamiento? La dimensión simbólica de la desigualdad social”. Trabajo y Sociedad 17:49-65.

Kay, C. 2005. "Enfoques sobre el Desarrollo Rural en América Latina y Europa desde mediados del Siglo Veinte". Ponencia presentada en el Seminario Internacional "Enfoques y perspectivas de la enseñanza del Desarrollo Rural", 31 de agosto al 2 de septiembre, Bogotá.

Long, N. 2007. Sociología del desarrollo: una perspectiva centrada en el actor. México: El Colegio de San Luis-CIESAS.

Moen, P. y E. Wethington 1992. "The concept of family adaptive strategies." Annual Review of Sociology 18:233-251.

Niederle, P. y C. Grisa 2008. "Diversificaçâo dos meios de vida e acceso a atores e ativos: uma abordagem sobre a dinámica de desenvolvimento local da agricultura familiar." Cuadernos de Desarrollo Rural 5:41-69.

Noe E. y H. Alroe 2003. "Farm enterprises as self-organizing systems: a new transdisciplinary framework for studying farm enterprises?." International Journal of Sociology of Agriculture and Food 11:3-14.

(van der) Ploeg, J. D. 1993. "Rural sociology and the new agrarian question. A perspective from the Netherlands." Sociologia Ruralis 33:240-260.

(van der) Ploeg, J. 2007. "Resistance of the third kind". Ponencia presentada en la European Rural Sociology Conference, 23 de agosto, Wageningen.

(van der) Ploeg, J. 2008. Camponeses e impérios alimentares. Lutas por autonomia e sustentabilidade na era da globalizaçao. Porto Alegre: UFRGS Editora.

(van der) Ploeg, J. 2010. "Farming Styles research: the state of the art". Ponencia presentada en el Workshop "Historicising farming styles", octubre. Melk.

(van der) Ploeg, J. D., J. Bouma, A. Rip, F. Rijkenberg, F. Ventura y J. Wiskerke 2004. "On regimes, novelties, niches and co-production". Pp. 1-30 in Seeds of transition, Essays on novelty production, niches and regimes in agriculture, edited by J. Wiskerke y J. D. van der Ploeg. Assen: Royal Van Gorcum Press.

Robeyns, I. 2003. The Capabilites Approach: An Interdisciplinary Introduction. Amsterdam: Department of Political Science and Amsterdam School of Social Sciences Research.

Rodríguez Bilella, P. y E. Tapella 2008. "Transformaciones globales, modos de vida y desarrollo rural". Pp 3-33 en Transformaciones globales y territorios. Desarrollo rural en Argentina. Experiencias y aprendizajes, editado por P. Rodríguez Bilella y E. Tapella. Buenos Aires: La Colmena.

Schneider, S. y P. Niederle 2008. "Agricultura familiar e teoría social: A diversidade das formas familiares de produçâo na agricultura". Pp. 989-1014 en Savanas: desafios e estratégias para o equilibrio entre sociedade, agronegócio e recursos naturais, editado por F. Faleiro y A. Farías Neto. Planaltina, D. F.: Embrapa Cerrados. 
Schneider, S. y P. Niederle 2010. "Resistance strategies and diversification of rural livelihoods: the construction of autonomy among Brazilian family farmers." Journal of Peasant Studies 37:379-405.

Scribano, A. 2009. Estudios sobre teoría social contemporánea: Baskhar, Bourdieu, Giddens, Habermas y Melucci. Buenos Aires: Ediciones CICCUS.

Scoones, I. 2009. "Livelihoods perspectives in rural development". Journal of Peasant Studies 36:171196.

Scott, J. 1987. "Resistance without protest and without organization: peasant opposition to the Islamic Zakat and the Cristian Tithe." Comparative Studies in Society and History 29: 417-452.

Scott, J. 1989. "Everyday forms of resistance", Copenhagen Papers in East and Southeast Asian Studies 4:33-62.

Scott, J. 2000. Los dominados y el arte de la resistencia. Discursos ocultos. México: Editorial Era.

Sen, A. 1981. Poverty and Famines: An Essay on Entitlement and Deprivation. Oxford: Oxford University Press.

Sewell, W. 1992. "A theory of structure: Duality, agency and transformation", American Journal of Sociology 98:1-29.

Sivaramakrishnan, K. 2005. "Some intellectual genealogies for the concept of everyday resistance". American Anthropologist 107:346-355.

Vanclay, F. L. Mesiti y P. Howden 1998. "Styles of farming and farming subcultures: Appropriate concepts for Australian rural sociology?.” Rural Society 9:85-107.

Vanclay, F., P. Howden, L. Mesiti y S. Glyde 2006. "The social and intellectual construction of farming styles: Testing Dutch ideas in Australian agriculture." Sociologia Ruralis 46:61-82.

Wilkis, A. 2004. "Apuntes sobre la noción de estrategia en Pierre Bourdieu." Revista Argentina de Sociología 2:118-130.

CLARA CRAVIOTTI es Doctora en Geografía de la Universidad de Buenos Aires y Licenciada en Sociología de la misma universidad. Se desempeña como investigadora de carrera del Consejo Nacional de Investigaciones Científicas y Técnicas (CONICET) y como docente de la Maestría de Estudios Sociales Agrarios de la Facultad Latinoamericana de Ciencias Sociales (FLACSO) de Argentina. Su campo de investigación es la sociología rural. Ha publicado los libros La otra agricultura. Trayectorias y estrategias de microemprendedores pampeanos; Los nuevos productores. Alimentos de alto valor y reestructuraciones agrarias; Entre el campo y la ciudad. Desafíos y estrategias de la pluriactividad en el agro; Azúcar y conflictos en el Norte argentino, y varios artículos en revistas académicas internacionales.

RECIBIDO: 06/09/2011

ACEPTADO: 20/04/2012

Publicado-online: 28/06/2012 